\title{
Investigations of the effect of viscosity of resin on the diffusion of pyrene in silicone polymer coatings using steady state fluorescence technique
}

\author{
C. Anandan, Bharathibai J. Basu *, K.S. Rajam \\ Surface Engineering Division, National Aerospace Laboratories, Bangalore 560017, India \\ Received 25 June 2003; received in revised form 25 June 2003; accepted 3 October 2003
}

\begin{abstract}
The diffusion phenomenon of pyrene in silicone coatings prepared from various commercial silicone resins with different viscosities was investigated using steady state excimer fluorescence technique. The amount of pyrene lost from the coatings by diffusion at different temperatures ranging from 25 to $70{ }^{\circ} \mathrm{C}$ was estimated from the excimer emission intensity. The diffusion coefficients $(D)$ and activation energy of diffusion of pyrene in silicone coatings were determined. It was found that the kinetic parameters of pyrene were comparable in all the four silicone coatings and independent of the viscosity of the resin. The $D$ values were lower than those expected for pyrene in PDMS resins in the corresponding viscosity range. This may be attributed to restricted mobility of pyrene due to cross-linking of polymer chains in the cured silicone coatings.
\end{abstract}

(C) 2003 Elsevier Ltd. All rights reserved.

Keywords: Pyrene; Silicone coatings; Diffusion coefficient; Fluorescence

\section{Introduction}

Silicone polymer coatings or films embedded with luminescent molecules are widely used for oxygen sensing applications [1-6]. Pyrene is often used as the fluorescent probe because it has a long excited state lifetime (400 ns) and high luminescence quantum yield and is efficiently quenched by oxygen [4-6]. Since pyrene has a vibronic intensity ratio that is highly sensitive to solvent polarity, it has been used as a potential tool for assessing the local environment in polymers [7]. The polymer support also has an active role in deciding the sensor performance. Silicone polymers are often preferred as the support matrix for oxygen sensors mainly because of their high oxygen permeability [8]. Optical sensors find

\footnotetext{
*Corresponding author. Tel.: +91-080-508-6251; fax: +91080-521-0113.

E-mail address: bharathi@css.cmmacs.ernet.in (B.J. Basu).
}

use not only for biological monitoring of oxygen but also for measuring surface pressure distribution for wind tunnel studies. The oxygen sensitive coating applied on the surface of a wind tunnel model for this purpose is known as pressure sensitive paint (PSP) and this specific application is referred as "luminescence barometry" [1-3].

We have been working on the development of PSPs, especially on the paints using pyrene as the pressure sensor and silicone polymer as the binder [9-12]. Even though pyrene-based PSPs have several advantages like high pressure sensitivity, low temperature coefficient and good mechanical properties, they lack stability and undergo steady degradation. We had carried out a detailed study of the mechanism of degradation of pyrene-based PSPs and found that the degradation of intensity of the PSP coating was due to evaporation losses of pyrene aided by diffusion of pyrene in silicone polymer [12]. Diffusion of pyrene was further accelerated by increase in temperature and decrease in pressure that are often encountered over the model surface during wind tunnel 
studies. This caused degradation of the emission intensity of the paint and resulted in inaccuracy of the surface pressure data. Hence it was clear that the diffusion of pyrene in the binder should be arrested in order to improve the stability of the PSP coatings. We have studied the nature of diffusion of pyrene molecules in pressure sensitive coatings under different experimental conditions of temperature and pressure as well as under storage conditions and the results obtained are described here.

Various fluorescence techniques such as steady state fluorescence or fluorescence quenching of certain additives to the polymers and in situ fluorescence of polymers end-capped with fluorescent labels have been used for the study of diffusion in polymers [13-18]. Guillet obtained values of diffusion coefficients of oxygen in different polymer matrix by luminescent quenching experiments [14]. Fluorescence method has also been used for monitoring diffusion of small molecules in polymer films $[14,15]$. Winnik et al. have studied cyclization dynamics of PDMS polymers end-capped on both ends with pyrene using a combination of fluorescence decay and steady state fluorescence measurements $[16,17]$. Even though all these works use the fluorescent molecules as probes, they do not deal with the diffusion of probe molecules themselves in the polymer matrix. Few studies on the diffusion rate of probe molecules in polymer supports are available $[14,15,18]$. For example, Chu and Thomas have studied the molecular mobility in poly dimethyl siloxane (PDMS) films by calculating the diffusion coefficient $(D)$ of pyrene using the measured rate constants and Smoluchowski equation [18]. We have used the decrease in excimer emission intensity as a measure of loss of pyrene by diffusion out of thin silicone coatings at ambient temperature and also at higher temperatures. Since it was necessary to decrease the diffusion rate of pyrene in the binder to improve the stability of the PSP coatings, the effect of viscosity of the silicone resin on the diffusion of pyrene in the PSP coating was studied. The $D$ values of pyrene were then calculated at different temperatures by applying onedimensional Fick's law of diffusion $[19,20]$. In the present study, diffusion coefficients and activation energy for diffusion for pyrene in silicone PSP coatings prepared from various commercial silicone resins with different viscosities were determined and compared.

\section{Experimental}

\subsection{Materials and polymer coating preparation}

Pyrene was procured from Acros Organics, Belgium and the four silicone prepolymers, RTV 615, RTV 11, RTV 420 and TSE 3455TA were purchased from G E Silicones, USA. All the prepolymers were commercial two-component silicone resins and their product data is given in Table 1. The reference luminophore, $\mathrm{Y}_{2} \mathrm{O}_{2} \mathrm{~S}: \mathrm{Eu}$ (red phosphor, type QKL63/N-C1) was procured from $\mathrm{M} / \mathrm{s}$ Phosphor Technology, UK. This compound was used as reference luminophore in the binary PSP coating. The emission intensity of this reference luminophore is proportional to the excitation intensity and hence this was used as an internal reference to normalize the emission intensity of pyrene and thus to correct the data for the fluctuations in the incident excitation radiation. Toluene (sulfur free) was used as the diluent for preparing the silicone resin solutions.

Silicone polymer coatings with $10 \mathrm{mM}$ pyrene were prepared from a mixture of $1 \mathrm{~g}$ of the silicone resin with $1 \mathrm{ml}$ of $10 \mathrm{mM}$ pyrene solution in toluene, $4 \mathrm{ml}$ toluene and $0.2 \mathrm{~g}$ red phosphor. This mixture was sprayed onto $150 \mathrm{~mm} \times 50 \mathrm{~mm}$ aluminum sheets of $0.3 \mathrm{~mm}$ thickness coated with a white reflecting undercoat [9,10]. This undercoat known as screen layer was prepared by spraying a two-component white epoxy paint onto clean and dry aluminum sheets and curing for $12 \mathrm{~h}$ at room temperature. The pyrene-silicone resin mixture was allowed to cure for $24 \mathrm{~h}$ at room temperature. Five sample coupons of size $50 \mathrm{~mm} \times 30 \mathrm{~mm}$ were cut from each aluminum sheet sprayed with each silicone resin mixture. The thickness of the coating was about $35 \pm 5 \mu \mathrm{m}$. An external reference coating was prepared in a similar manner by spraying a mixture of $1 \mathrm{~g}$ silicone resin with $0.2 \mathrm{~g}$ red phosphor in toluene.

\subsection{Methods}

\subsubsection{Fluorescence measurements}

Fluorescence emission spectra were recorded using a fiber optic spectrometer, model SD1000 from Ocean Optics, Inc USA and a $300 \mathrm{~W}$ Xenon Arc Lamp (model no. 6258 from Oriel Instruments) as source. The infrared radiation from the xenon lamp was filtered by means of

Table 1

Product data and properties of different silicone resins and cured silicone polymer

\begin{tabular}{lcllll}
\hline Name & $\begin{array}{l}\text { Viscosity } \\
(\mathrm{cP})\end{array}$ & $\begin{array}{l}\text { Operating temperature } \\
\text { range }\left({ }^{\circ} \mathrm{C}\right)\end{array}$ & $\begin{array}{l}\text { Hardness } \\
\text { (Shore A) }\end{array}$ & $\begin{array}{l}\text { Specific } \\
\text { gravity }\end{array}$ & $\begin{array}{l}\text { Elongation } \\
(\%)\end{array}$ \\
\hline RTV 615 (addition) & 4000 & -60 to 204 & 44 & 1.02 & 120 \\
RTV 11 (condensation) & 11,000 & -60 to 204 & 41 & 1.19 & 190 \\
RTV 420 & 30,000 & -30 to 200 & 20 & 1.24 & 600 \\
TSE 3455TA & 45,000 & -30 to 200 & 40 & 1.10 & 360 \\
\hline
\end{tabular}


a water filter. A bandpass filter (Oriel cat. no. 51650) was used to transmit UV radiation from the source in the wavelength range of $300-380 \mathrm{~nm}$ and to illuminate the coatings with UV radiation. The coatings were mounted in a sample chamber fabricated in our laboratory so that emission was measured by the front face technique.

\subsubsection{Annealing experiments}

Annealing experiments of the coatings were carried out in a temperature controlled vacuum oven in which 0.1 bar pressure can be obtained using a rotary vacuum pump. The temperature can be controlled to $\pm 1{ }^{\circ} \mathrm{C}$ as indicated by a calibrated thermometer. Sample coatings were annealed at selected temperatures between 50 and $75{ }^{\circ} \mathrm{C}$ for different intervals of time. After each annealing step, the coatings were cooled to room temperature and fluorescence spectra were recorded. The loss of pyrene from the coatings due to diffusion was estimated by measuring the decrease in excimer emission intensity of the coating.

It was found that the red emission from the internal reference luminophore present in the silicone coatings increased after each annealing step. This was due to the decrease in pyrene concentration in the coating after annealing. As the incident light intensity was kept constant, this increase in red emission from the internal reference luminophore can lead to systematic errors in the analysis. Therefore, for aging studies at room temperature, where measurements were spread over several days/weeks, the blue intensity was normalized with respect to red counts from an external reference coating.

\section{Results and discussion}

\subsection{Diffusion of pyrene in silicone polymer coatings at ambient temperature}

The loss of pyrene from silicone polymer coatings under storage conditions at room temperature and pressure was determined by measuring the blue emission intensity at periodic intervals. Fig. 1 shows the fluorescent emission spectrum of a typical pyrene-based binary PSP coating recorded at ambient temperature and atmospheric pressure. The broad emission peak at 475 $\mathrm{nm}$ is due to pyrene excimer emission and this is sensitive to oxygen partial pressure and hence to variations in air pressure. The narrow emission peak at $626 \mathrm{~nm}$ is due to the reference luminophore and this peak is insensitive to oxygen and is sensitive only to variations in the excitation intensity. The fluorescence emission spectrum of a reference coating also was recorded to apply corrections for the slight variations in the excitation intensity on different days. The blue emission $\left(I_{\text {blue }}\right)$ of the sample coatings and the red emission $\left(I_{\text {ref }}\right)$ of the reference

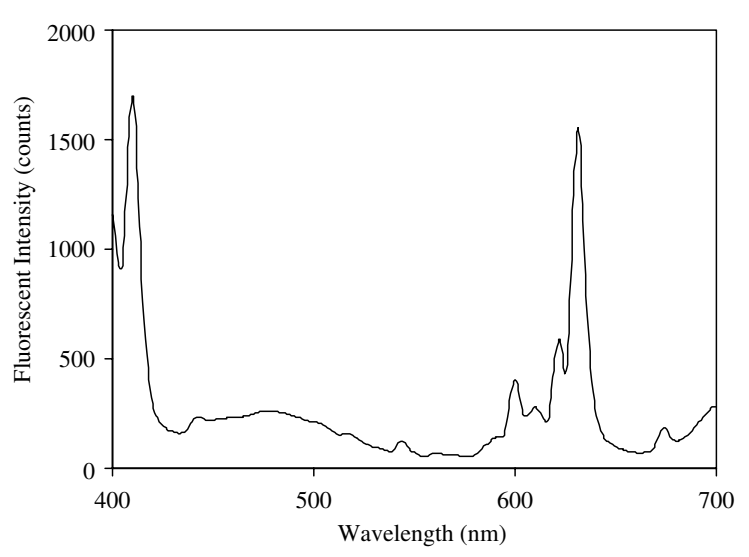

Fig. 1. Fluorescent emission spectrum from a typical pyrenebased binary PSP coating at ambient temperature and atmospheric pressure.

coating were measured. The blue intensities were normalized with reference to the red counts using the equation, $\left(I_{\text {blue }} / I_{\text {ref }}\right) \times 100$.

The first measurement was done after complete curing of the paint. Generally complete curing of the coatings takes place in $24 \mathrm{~h}$ for RTV silicone resins. Subsequent measurements were done at short intervals of one or two days. Fig. 2 shows the variation of blue emission of pyrene (normalized with reference to the red emission from the reference coating) from different silicone coatings as a function of storage time in days. As aging progressed, there was a steady loss of pyrene from the coating due to evaporation and diffusion of pyrene at room temperature. As a result of this, the blue intensity showed a steady decrease with time. This decrease in emission intensity was related to the amount of pyrene lost from the coating in the following way.

Since the thickness of the silicone coating is in the range of few tens of microns, one-dimensional Fick's second law of diffusion was applied for analyzing the data with the assumption that the diffusion coefficient of

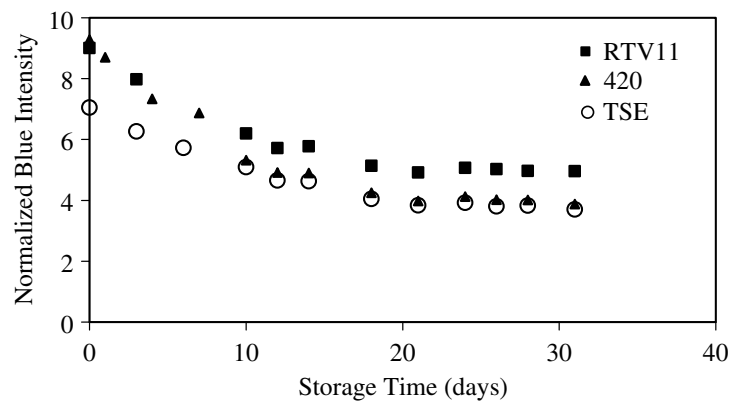

Fig. 2. Variation of normalized blue intensity from the three coatings with storage time in days under ambient conditions. 
pyrene is independent of its concentration $[19,20]$. In addition to this, it was further assumed that

(i) The excimer emission intensity is directly proportional to the concentration of pyrene in the coating. Our earlier experimental results have shown that this is valid at pyrene concentrations from 3 to 15 $\mathrm{mM}$ in the coating, i.e., when concentration quenching is absent. Hence coatings with $10 \mathrm{mM}$ pyrene concentration were selected for aging studies.

(ii) The fraction of excimer fluorescence is constant at all pyrene concentrations chosen for this study. As the aging proceeds, the pyrene concentration in the coating decreases and at very low concentrations, the excimer emission becomes negligible and the monomer emission becomes predominant. This may happen at pyrene concentrations $<1 \mathrm{mM}$.

With appropriate initial $(t=0)$ and boundary conditions, one can solve the diffusion equation to get an analytical solution. In the present case, the sample is a thin silicone coating of thickness $L$ containing $10 \mathrm{mM}$ pyrene over a white epoxy undercoat. It is assumed that pyrene molecules are uniformly distributed within the silicone coating. The boundary conditions are determined at the coating surfaces. It has been reported earlier that the diffusion of pyrene in the epoxy undercoat is negligible $[12,18]$. Therefore, pyrene molecules that diffuse and reach the interface between the epoxy coating and the silicone coating diffuse back into the latter. Thus diffusion takes place only towards the surface of the silicone coating. Pyrene molecules that reach the surface of the silicone coating are desorbed from the surface. With these initial and boundary conditions one can obtain an analytical solution for the loss of pyrene with time by diffusion.

As we have used only the initial part of the intensity vs. time curve for the analysis, the approximate form of the solution to the one-dimensional diffusion equation is used for analyzing the experimental data.

$\Delta M=\left(C_{t}-C_{0}\right) /\left(C_{\infty}-C_{0}\right)=(4 / L)(D t / \pi)^{1 / 2}$

where $\Delta M$ is the fractional mass loss, $C_{0}$ is the initial pyrene concentration, $C_{t}$ and $C_{\infty}$ respectively are the pyrene concentrations after a lapse of time $t$ and after infinitely long time.

If $I_{0}, I_{t}$ and $I_{\infty}$ respectively are the emission intensities before aging, after aging for time $t$ and after aging for a long time till there was no further mass loss, then the fractional intensity decrease is given by the following equation,

$\Delta I=\left(I_{0}-I_{t}\right) /\left(I_{0}-I_{\infty}\right)$

As discussed above, since the excimer fluorescence intensity of pyrene is proportional to the pyrene concentration in the coating, Eq. (1) can be written as
$\Delta M=\left(I_{0}-I_{t}\right) /\left(I_{0}-I_{\infty}\right)=(4 / L) \sqrt{ }(D t / \pi)$

The fractional loss of pyrene, $\Delta M$ is plotted as a function of square root of time. The diffusion coefficient, $D$ is then given by

$D=(1 / 16) \pi L^{2} s^{2}$

where $s$ is the slope of the linear portion of the plot. The diffusion coefficient, $D$ can be estimated using Eq. (4) [20].

Since diffusion is an activated process, its temperature dependence is given by

$D=D_{0} \exp \left(E_{\mathrm{A}} / k T\right)$

where $E_{\mathrm{A}}$ is the activation energy of diffusion, $k$ is the Boltzmann constant, and $D_{0}$ is the pre-exponential factor. The activation energy of diffusion can be determined by obtaining $D$ values at different temperatures and using the Arrhenius' plot $\ln (D)$ vs. $1 / T$.

The fractional mass loss of pyrene, $\Delta M$ was calculated from the fractional intensity decrease as per Eq. (3). The variation of $\Delta M$ from three of the silicone coatings prepared with RTV 11, RTV420 and TSE $3455 \mathrm{TA}$ as a function of storage time is shown in Fig. 3. It can be seen that the rate of loss of pyrene was similar in all the three coatings irrespective of the difference in their composition. $\Delta M$ is plotted as a function of square root of time in Fig. 4. The data plotted in this way shows the typical behavior of absorption/desorption of molecules from polymer films limited by diffusion. The data for the initial period of diffusion up to a mass loss of $60 \%$ for the three coatings were fitted by the linear least square method. The diffusion coefficients of pyrene at room temperature in the three silicone coatings, RTV 420, RTV 11 and TSE 3455T were calculated using Eq. (4) and were found to be $2.2 \times 10^{-12}, 2.5 \times 10^{-12}$ and $5.7 \times 10^{-12} \mathrm{~cm}^{2} / \mathrm{s}$ respectively.

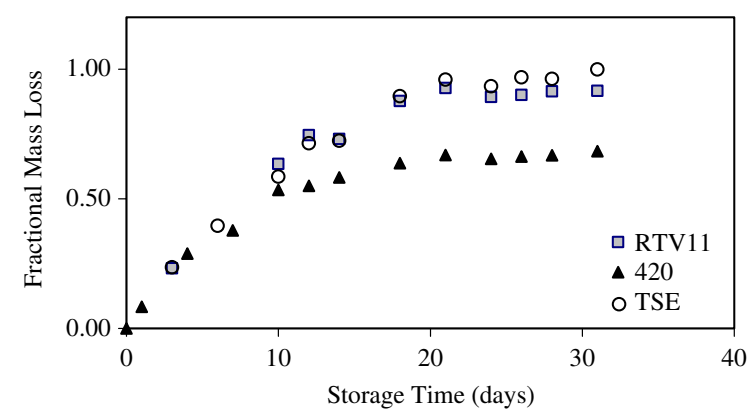

Fig. 3. Variation of fractional mass loss of pyrene from the three coatings with storage time under ambient conditions. 


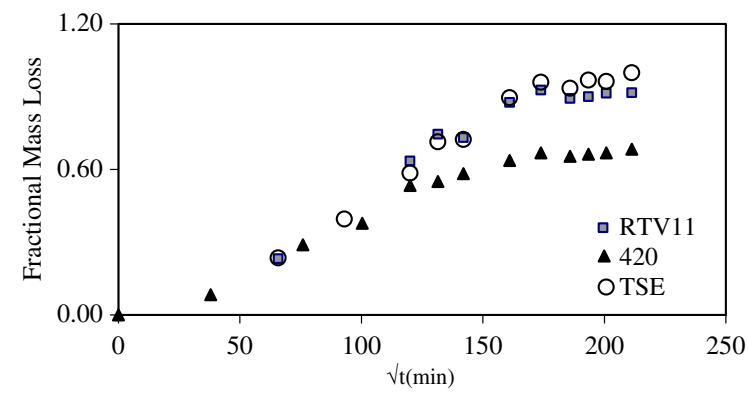

Fig. 4. Fractional mass loss of pyrene from the three coatings as a function of square root of storage time in minutes.

\subsection{Diffusion of pyrene in silicone polymer coatings at higher temperatures}

The loss of pyrene from silicone polymer coatings due to annealing at different temperatures and 0.1 bar was determined by measuring the blue emission intensity at periodic intervals. Fig. 5 shows the variation of normalized blue emission with annealing time for a typical silicone coating prepared with RTV 615 at different temperatures. It was found that the decrease in intensity was higher at higher annealing temperatures. The $\Delta M$ values were obtained after each annealing step at different temperatures for the coatings. In a similar way, $\Delta M$ values were determined for the other three resins, namely RTV 11, RTV 420 and TSE 3455T. These were

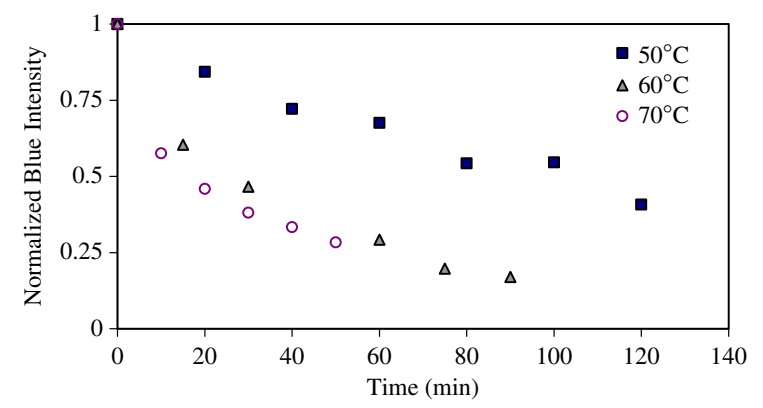

Fig. 5. Change of normalized blue intensity from a typical coating prepared with RTV 615 with annealing time at three different temperatures.

plotted as a function of square root of annealing time and these are shown in Fig. 6(a)-(d). The data were fitted by the linear least square method and the diffusion coefficients at different annealing temperatures were estimated from the slopes of the plots. The diffusion coefficients for pyrene in the four silicone coatings obtained at different temperatures are given in Table 2. It can be seen from Table 2 that the diffusion coefficient increases with increase in temperature.

\subsection{Determination of activation energy for diffusion}

The diffusion coefficients of pyrene in different silicone coatings at different temperatures were estimated
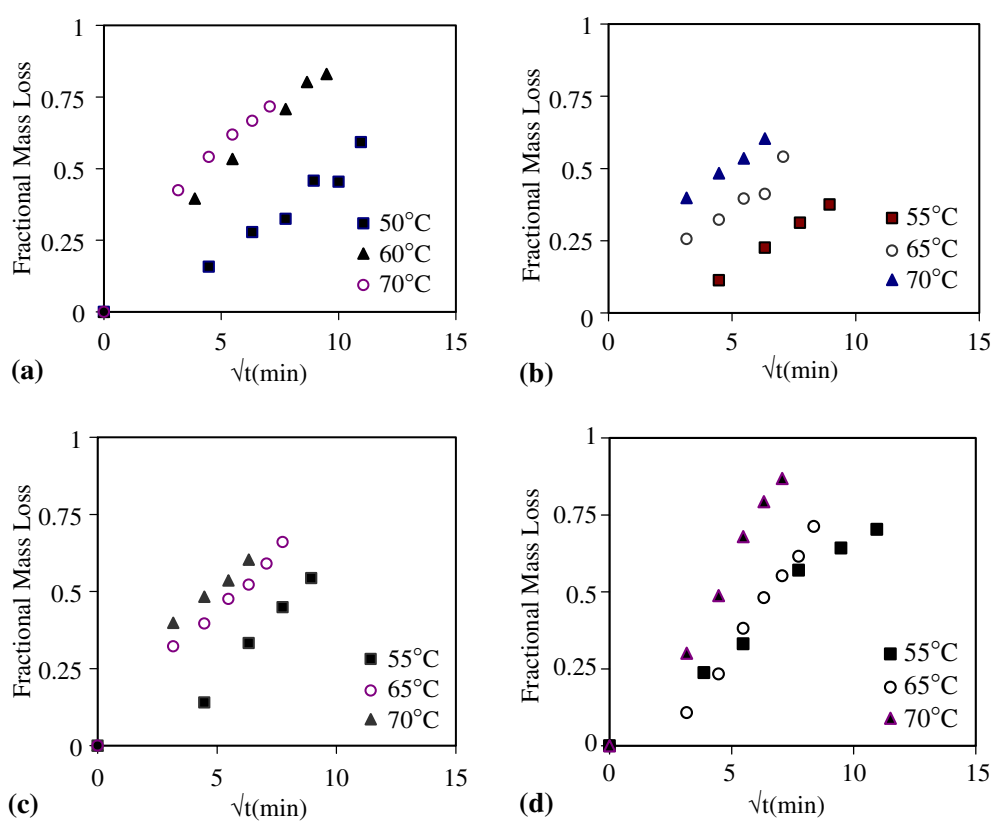

Fig. 6. Fractional mass loss of pyrene from the three coatings in all the four coatings as a function of square root of annealing time at different temperatures. (a) RTV 615, (b) RTV 11, (c) RTV 420 and (d) TSE 3455T. 
Table 2

Effect of temperature on the diffusion coefficient of pyrene in different silicone coatings

\begin{tabular}{lllcl}
\hline Temperature $\left({ }^{\circ} \mathrm{C}\right)$ & $\begin{array}{l}\text { RTV } 615 D \times 10^{10} \\
\left(\mathrm{~cm}^{2} / \mathrm{s}\right)\end{array}$ & $\begin{array}{l}\text { RTV } 11 D \times 10^{10} \\
\left(\mathrm{~cm}^{2} / \mathrm{s}\right)\end{array}$ & $\begin{array}{l}\text { RTV } 420 D \times 10^{10} \\
\left(\mathrm{~cm}^{2} / \mathrm{s}\right)\end{array}$ & $\begin{array}{l}\text { TSE 3455 T } D \times 10^{10} \\
\left(\mathrm{~cm}^{2} / \mathrm{s}\right)\end{array}$ \\
\hline 25 & - & 0.0215 & 0.016 & 0.057 \\
50 & 0.84 & - & - & - \\
55 & - & 1.17 & 1.6 & 1.30 \\
60 & 2.32 & 1.52 & 1.03 & 2.43 \\
65 & 2.75 & 2.1 & 4.8 & 5.08 \\
70 & 4.96 & 4.13 & 12.83 & 5.72 \\
75 & - & - & - & \\
\hline
\end{tabular}
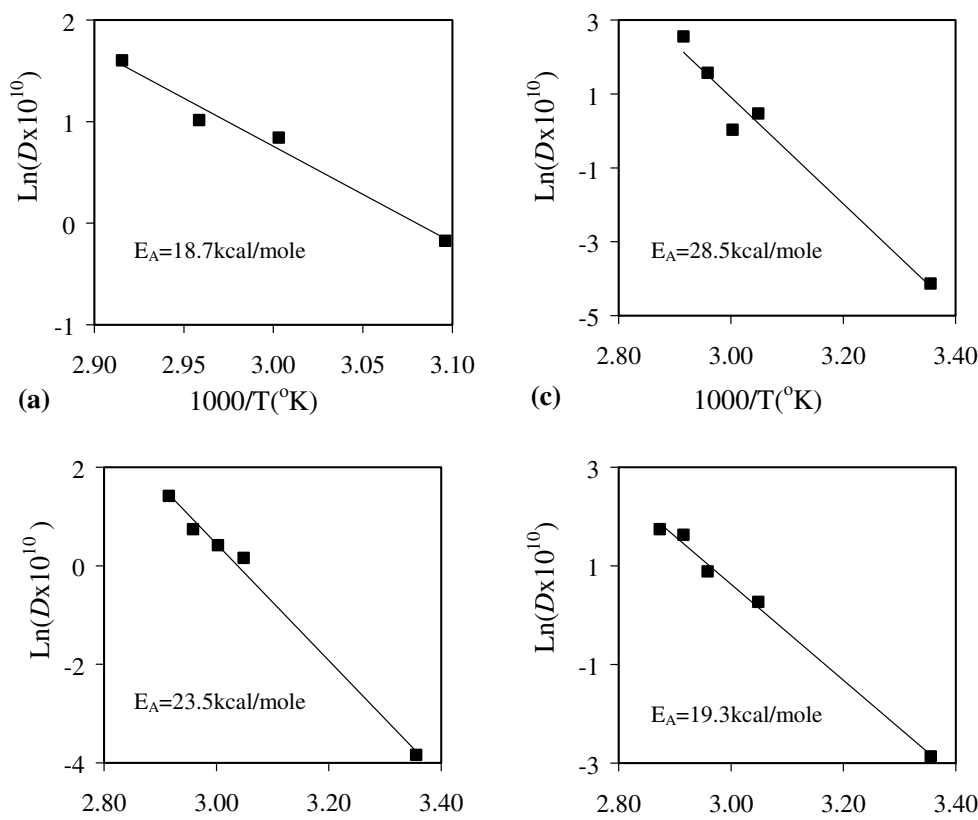

(b)

$1000 / \mathrm{T}\left({ }^{\circ} \mathrm{K}\right)$

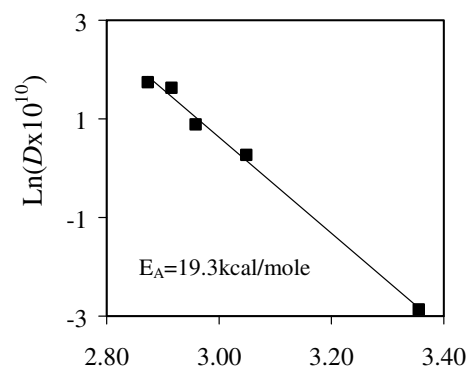

(d) $\quad 1000 / \mathrm{T}\left({ }^{\circ} \mathrm{K}\right)$

Fig. 7. Arrhenius' plot for diffusion of pyrene in all the four coatings. (a) RTV 615, (b) RTV 11, (c) RTV 420 and (d) TSE $3455 T$.

Table 3

Diffusion data for pyrene in cured silicone coatings prepared with resins of different viscosities

\begin{tabular}{lclll}
\hline Name & Viscosity $(\mathrm{cP})$ & $D_{\text {expt }} \times 10^{10}\left(\mathrm{~cm}^{2} / \mathrm{s}\right)^{\mathrm{a}}$ & $D_{\text {Stokes }} \times 10^{10}\left(\mathrm{~cm}^{2} / \mathrm{s}\right)^{\mathrm{b}}$ & $E_{\mathrm{A}}(\mathrm{kcal} / \mathrm{mole})$ \\
\hline RTV 615 (addition) & 4000 & $0.088^{\mathrm{c}}$ & 23.2 & 18.81 \\
RTV 11 (condensation) & 11,000 & 0.025 & 8.45 & 24.32 \\
RTV 420 & 30,000 & 0.022 & 3.1 & 27.56 \\
TSE 3455TA & 45,000 & 0.057 & 2.07 & 19.13 \\
\hline
\end{tabular}

${ }^{\text {a }} D_{\text {expt }}$ is the experimentally determined diffusion coefficient at room temperature.

${ }^{\mathrm{b}} D_{\text {Stokes }}$ is calculated from Stokes-Sutherland equation, Eq. (7) with $r=3.46 \AA$ and $k=1.38 \times 10^{-16} \mathrm{erg} / \mathrm{K}$.

${ }^{\mathrm{c}}$ Calculated value using the experimentally determined activation energy and $D$ at $50{ }^{\circ} \mathrm{C}$ and Eq. (5).

and $\ln (D)$ was plotted vs. $1000 / T$. The $\ln (D)$ vs. $1000 / T$ plots for the different coatings are shown in Fig. 7(a)(d). The activation energy of diffusion, $E_{\mathrm{A}}$ of pyrene in different silicone polymers was calculated from the slope of these plots after fitting the data by a least square fit. The $E_{\mathrm{A}}$ values are given in Table 3 . It can be seen that the activation energy lies in the range 19-29 kcal/mole for the four silicone coatings. 


\subsection{Mechanism of diffusion of pyrene in silicone polymer coatings}

For a spherical molecule moving in a viscous medium, the diffusion coefficient, $D$ is related to the viscosity of the medium, $\eta$ by the Stokes-Einstein equation

$D=k T / 6 \pi r \eta$

where $k$ is the Boltzmann's constant, $T$ is the temperature and $r$ is the radius of the moving particle [21]. Sutherland's correction for a small molecule moving in a medium of large molecule leads to the following form of the above formula

$D=k T / 4 \pi r \eta$

This equation shows that the diffusion coefficient decreased as viscosity of the medium increased.

Table 3 gives the experimentally determined diffusion coefficients at room temperature in the silicone coatings under study and also the $D$ values calculated using Eq. (7) in which $r$, the interaction radius of pyrene for excimer formation is taken as $3.46 \AA$ [18]. As can be seen from Table 3, the experimentally determined diffusion coefficient changed only slightly when the viscosity of the resins was varied from 4000 to $45,000 \mathrm{cP}$. These results showed that $\eta$ has marginal influence on $D$. Similar non-Stokian observations have been reported earlier [13,18,21]. Cox and Dunn have measured the fluorescence emission intensity of 9,10-diphenyl antharacene probe molecules in PDMS liquids with viscosity in the range $0.65-12,500 \mathrm{cS}$ and found the emission intensity to increase for $1 \leqslant \eta(\mathrm{cS}) \leqslant 10^{3}$ [13]. At higher viscosities $\left(\eta>10^{3} \mathrm{cS}\right)$ no further increase in emission intensity was found. A non-Stokes-Einstein dependence of diffusion coefficient on viscosity of the fluid medium has been reported earlier for several solute-solvent systems [21].

In a study of molecular mobility in polymers, Chu and Thomas examined dynamic excimer formation of pyrene in PDMS in thin films and bulk PDMS with viscosities in the range of $1-2.5 \times 10^{6} \mathrm{cP}$ at $20^{\circ} \mathrm{C}$ [18]. They calculated the diffusion coefficient of pyrene using the excimer decay rate and the Smoluchowski's equation for bimolecular reaction and compared it with that obtained by the Stokes-Sutherland equation given above. Their results showed $D$ to be nearly independent of $\eta$ for $\eta>50 \mathrm{cP}$ and experimentally determined $D$ values were much higher than that determined from the above equation. This discrepancy was explained by them on the basis that the local or the "microviscosity" being much lower than the macro viscosity. Guillet has discussed the role of microviscosity and macro viscosity in polymers and recommends the use of microviscosity in interpreting transport phenomena in polymers [15]. According to the free volume theory the side group rotation and segmental motions in polymer chains create enough holes or free volume for the small molecules to diffuse through. Thus the viscosity encountered by the molecules on this microscopic scale, the "microviscosity" is much lower than the macro viscosity due to the bulk motion of the polymer chains as a whole. Hence the experimental diffusion coefficients in such systems are higher than the calculated values.

Our experimental diffusion coefficients for pyrene in silicone coatings are much lower than those reported for pyrene in PDMS resins in the corresponding viscosity range. The lower $D$ values may be due to the restricted mobility of pyrene molecules in the cured silicone coatings as used in this study. Unlike the fluorescence excimer decay method, a transient technique, wherein the pyrene molecules have to diffuse $3.46 \AA$, the distance to form the excimers, in steady state fluorescence intensity method used by us the pyrene molecules have to diffuse much longer distances. This distance is equal to the thickness of the coatings, several microns, reflecting "diffusion on a macroscopic scale". This implies that "macro scale diffusion" is also assisted by the same mechanism that assists the pyrene diffusion at much lower length scale i.e. side group rotation and segmental motion of the polymer chain at a reduced speed.

The temperature dependence of $D$ was interpreted by assuming that the diffusion obeys Arrhenius relation. The activation energy lies in the range 19-28 kcal/mole. For the side group rotation in polymers the activation energy is in the range of $16-35 \mathrm{kcal} / \mathrm{mole}$ [22]. Our values are closer to this. This further confirms that both the microscale diffusion and macro scale diffusion are affected by the same mechanism.

Two of the resins used in the present study, RTV 11 and RTV 420 contained filler materials added to them. RTV 11 had fumed silica as filler but the nature of filler in RTV 420 is not clear. Further, all the four coatings contained a reference luminophore (an inorganic material) as a reference to apply corrections for incident light fluctuations. The diffusion and quenching phenomena in the presence of fillers such as silica has been studied in the past in several solute-polymer systems. Recently Lu and Winnik have reviewed the role of filler materials in polymer binders used for oxygen sensors [23]. They had shown that the effect of filler on the response of the system depends on the nature of interactions between the diffusing species and the filler material. If the interaction is weak then the filler will act merely as an obstacle in the path of the diffusing species decreasing the diffusion coefficient. It has been reported that the interaction of pyrene with filler materials like silica gel is very weak and it diffuses on the filler surfaces with a slight hindrance [24]. Therefore, in the present work, the diffusion coefficients in silicone coating with fillers may be taken to be slightly lower than that without filler material. 


\section{Conclusions}

The diffusion of pyrene in silicone polymer coatings prepared from various commercial silicone resins with different viscosities was studied using steady state excimer fluorescence of pyrene. This study has established that pyrene diffuses in all of them nearly at the same rate irrespective of the difference in their composition or the viscosity of the resin. The diffusion coefficient $(D)$ and activation energy of diffusion of pyrene in these coatings were determined and compared. The kinetic parameters were comparable in all the four silicone coatings. This indicated that the viscosity of the resins have marginal effect on the diffusion of pyrene in the cured polymer. The diffusion of pyrene was accelerated by increase in temperature. The high diffusion rate of pyrene in the cured coatings is attributed to the side group rotation of the PDMS chains. But the $D$ values were lower than those reported for pyrene in PDMS resins in the corresponding viscosity range. The lower $D$ values in coatings may be attributed to the restricted mobility of pyrene due to cross-linking in the cured silicone polymer. Even with this restricted mobility, the diffusion coefficients were higher than that predicted by Stokes-Sutherland equation.

\section{Acknowledgements}

The authors would like to thank Director, N.A.L. for his support and permission to publish the work. Our thanks are also due to A.R. Dinesh and A. Thirumurugan for their help in sample preparation and measurements.

\section{References}

[1] Demas JN, Degraff BA, Coleman PB. Oxygen sensors based on luminescence quenching. Anal Chem 1999;71: 793A-800A.

[2] Gouterman M. Oxygen quenching of luminescence of pressure sensitive paint for wind tunnel research. J Chem Educ 1997;74(6):697-702.

[3] Bell JH, Schairer ET, Hand LA, Mehta RD. Surface pressure measurements using luminescence coatings. Ann Rev Fluid Mech 2001;33:155-206.

[4] Lubbers DW, Optiz N. Optical fluorescence sensors for continuous measurement of chemical concentrations in biological systems. Sens Actuators 1983;4:641-54.

[5] Lee ED, Werner TC, Seitz WR. Luminescence ratio indicators for oxygen. Anal Chem 1987;59:279-83.

[6] Xu W, McDonough RC, Langsdorf B, Demas JN, Degraff BA. Oxygen sensors based on luminescence quenching:
Interactions of pyrene with the polymer supports. Anal Chem 1995;67:3172-80.

[7] Kalyanasundaram K, Thomas JK. Environmental effects on the vibronic band intensities in pyrene monomer fluorescence and their applications in studies of micellar systems. J Am Chem Soc 1977;99:2039-44.

[8] Bandrup J, Immergut EH, editors. The polymer handbook. 3rd ed. New York: Wiley; 1989.

[9] Basu BJ, Kannan R, Rajagopal I, Rajam KS. Development and evaluation of the first binary pressure sensitive paint NAL-G. NAL Project Document SE 0104; 2001.

[10] Basu BJ, Anandan C, Rajam KS. Investigations on the development of improved binary pressure sensitive paint NAL-G1. NAL Project Document SE 0112; 2001.

[11] Basu BJ, Anandan C, Rajam KS. Development and evaluation of the improved binary pressure sensitive paint NAL-G2. NAL Project Document SE 0113; 2001.

[12] Basu BJ, Anandan C, Rajam KS. Study of the mechanism of degradation of pyrene-based pressure sensitive paint. Sens Actuators B 2003;94:257-66.

[13] Cox ME, Dunn B. Detection of oxygen by fluorescence quenching. Appl Opt 1985;24:2114-20.

[14] Guillet JE. In: Winnik MA, editor. Photophysical and photochemical tools in polymer science. Dordrecht, The Netherlands: Reidel; 1986. p. 467-94.

[15] Guillet JE. Polymer photophysics and photochemistry. Cambridge University Press; 1985.

[16] Svirskaya P, Danhelka J, Redpath AEC, Winnik MA. Cyclization dynamics of polymers: 7. Applications of the pyrene excimer technique to the internal dynamics of polydimethylsiloxane chains. Polymer 1983;24:31822.

[17] Winnik MA, Redpath AEC, Svirskaya P, Mar A. The influence of polymer concentration on the internal motion-intramolecular pyrene excimer formation - of a low molecular weight probe in solution. Polymer 1983;24:473-5.

[18] Chu DY, Thomas DK. Photophysical studies of molecular mobility in polymer films and bulk polymers. 3. Dynamic excimer formation of pyrene in bulk PDMS. Macromolecules 1990;23:2217-22.

[19] Crank J. Mathematics of diffusion. London: Oxford University Press; 1956.

[20] Crank J, Park GS. Diffusion in polymers. New York: Academic Press; 1968. p. 1-39.

[21] Alwattar AH, Lumb MD, Birks JB. Diffusion-controlled rate processes, vol. I. In: Birks JB, editor. Organic molecular photophysics. Wiley-Interscience; 1973. p. 403-54 (Chapter 8).

[22] Hedvig P. Dielectric spectroscopy of polymers. Bristol: Adam Hilger Ltd; 1977.

[23] Lu X, Winnik MA. Luminescence quenching in polymer/ filler nanocomposite films used in oxygen sensors. Chem Mater 2001;13:3449-63.

[24] Krasnansky R, Thomas JK. Diffusional vs. non-diffusional fluorescence quenching at the silica gel liquid-solid and vacuum-solid interfaces: pyrene fluorescence quenching by oxygen, nitromethane and nitropropionic acid. J Photochem Photobiol A: Chem 1991;57:81-96. 